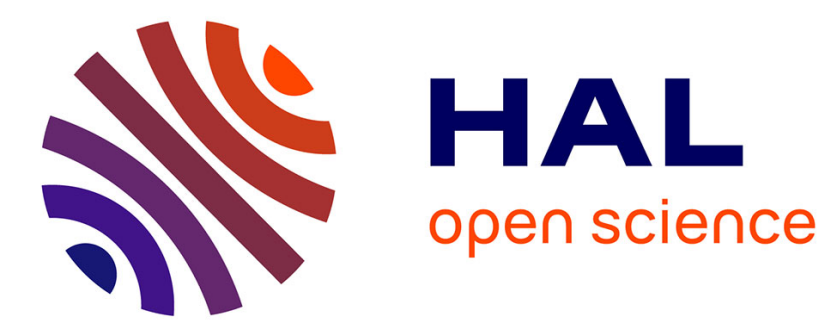

\title{
L-embedded Banach spaces and a weak version of Phillips lemma
}

\author{
Hermann Pfitzner
}

\section{To cite this version:}

Hermann Pfitzner. L-embedded Banach spaces and a weak version of Phillips lemma. Vector Measures, Integration and Applications, Sep 2008, Eichstätt, Germany. pp.339-344. hal-00463783

\section{HAL Id: hal-00463783 \\ https://hal.science/hal-00463783}

Submitted on 15 Mar 2010

HAL is a multi-disciplinary open access archive for the deposit and dissemination of scientific research documents, whether they are published or not. The documents may come from teaching and research institutions in France or abroad, or from public or private research centers.
L'archive ouverte pluridisciplinaire HAL, est destinée au dépôt et à la diffusion de documents scientifiques de niveau recherche, publiés ou non, émanant des établissements d'enseignement et de recherche français ou étrangers, des laboratoires publics ou privés. 


\title{
L-embedded Banach spaces and a weak version of Phillips lemma
}

\author{
Hermann Pfitzner
}

\begin{abstract}
After a short survey on L-embedded Banach spaces - for which Bochner spaces of the form $L^{1}(X)$ with reflexive $X$ serve as examples - we prove that these spaces satisfy a weak form of Phillips' lemma: It is proved that the L-projection of an L-embedded Banach spaces sends relatively $w^{*}$ sequentially compact sets to relatively weakly sequentially compact sets.
\end{abstract}

Mathematics Subject Classification (2000). 46B20.

Keywords. L-embedded, L-projection, Phillips lemma, Bochner spaces.

L-embedded Banach spaces. A projection $P$ on a Banach space $Z$ is called an $L$-projection if $\|P z\|+\|z-P z\|=\|z\|$ for all $z \in Z$. A Banach space $X$ is called $L$-embedded (or an L-summand in its bidual) if it is the image of an L-projection on its bidual. In this case we write $X^{* *}=X \oplus_{1} X_{\mathrm{s}}$. The standard reference for L-embedded Banach spaces is the monograph [3] which contains everything of this introduction except for [7]. For general Banach space theory and undefined notation we refer to [4], [6], or [1].

A special class of L-embedded spaces consists of the duals of M-embedded Banach spaces: A Banach space $Y$ is called $M$-embedded (or an M-Ideal in its bidual) if its annihilator $Y^{\perp}$ in $Y^{* * *}$ is the range of an L-projection on $Y^{* * *}$ in which case the dual $Y^{*}$ identifies easily with the kernel of the L-projection and is therefore L-embedded. Examples of such L-embedded spaces include $l^{1}(\Gamma)=\left(c_{0}(\Gamma)\right)^{*}(\Gamma$ any set), $\mathrm{N}(H)=(\mathrm{K}(H))^{*}$ (the nuclear operators on a Hilbert space $\left.H\right),\left(\mathrm{K}\left(l^{p}, l^{q}\right)\right)^{*}$ where $1<p \leq q<\infty$, the Hardy space $H_{0}^{1}=(C(\mathbb{\Gamma}) / \mathrm{A})^{*}$ where $A$ is the disk algebra.

$L^{1}$-spaces are L-embedded and so are, more generally, the preduals of von Neumann algebras, even the preduals of JBW*-triples. As to a generalization towards Bochner spaces of the form $L^{1}(X)$ not much seems to be known: From [3, p. 199] we resume the following cases. Since the projective tensor product of $L^{1}$ and the predual of von Neumann algebra is again the predual of a von Neumann algebra the Bochner space $L^{1}(X)$ is L-embedded if $X$ is a von Neumann predual. 
Further, if the underlying measure space is finite and if $X \subset L^{1}$ is (in Godefroy's terminology) nicely placed i.e. L-embedded, for example $H_{0}^{1} \subset L^{1}(\mathbb{\Gamma})$, then $L^{1}(X)$ is L-embedded, too. Finally, if $X$ is reflexive (hence trivially L-embedded) then $L^{1}(X)$ is L-embedded.

The class of L-embedded spaces is much larger than the one of duals of Membedded spaces because among $L^{1}$-spaces only $l^{1}(\Gamma)$ ( $\Gamma$ a set) is the dual of an M-embedded space, and, analogously, the only von Neumann predual which is the dual of an M-embedded space is the direct sum in the $l^{1}$-sense of $\mathrm{N}(H)$ spaces which in turn is equivalent to the von Neumann predual having RNP [3, Prop. III.2.9, Prop. IV.2.9]. In particular, an L-embedded space need not have RNP whereas the dual of an M-embedded space does. There are dual L-embedded spaces with RNP that are not duals of M-embedded spaces [3, Ex. IV.4.12].

The interest of L-embeddedness lies in the fact that it unifies some Banach space properties which before were known only as special cases. A kind of breakthrough for this general point of view was Godefroy's discovery that L-embedded Banach spaces are weakly sequentially complete. Another example of this unifying point of view is the fact [7] that (at least) separable L-embedded spaces are unique preduals which comprises for example the corresponding result of Dixmier-Sakai for von Neumann preduals.

Talagrand [10] has shown that the Bochner space $L^{1}(X)$ is weakly sequentially complete if and only if $X$ is and Randrianantoanina [9] has shown that $L^{1}(X)$ has Pełczyński's property $\left(\mathrm{V}^{*}\right)$ if and only if $X$ does (see also [5]). Since L-embedded spaces are weakly sequentially complete and have $\left(\mathrm{V}^{*}\right)[3, \mathrm{IV} .2 .7]$ it seems plausible that $L^{1}(X)$ is L-embedded for more Banach spaces $X$ than the ones mentionned above.

A weak version of Phillips' lemma. Phillips' lemma [8] states that the canonical projection from the second dual of $l^{1}$ onto $l^{1}$ is $w^{*}$-norm-sequentially continuous which if one takes $l^{1}$ 's Schur property for granted is equivalent to the projection being $w^{*}$-weak-sequentially continuous. (Cf., for example, [1, Ch. VII].) Therefore the fact that the canonical projection from the third onto the first dual of a Banach space is $w^{*}$-weak sequentially continuous if the space in consideration has Pelczyński's property (V) (cf. [3, Prop. III.3.6]) can be considered a generalization of Phillips' lemma.

In this note we give a modest - see the remark after the proof - generalization in a similar direction by looking at $l^{1}$ as an L-embedded Banach space. Although the result is new the technique of its proof is not. In fact it is a modification of the proof in [7] but holds, contrary to the latter one, for all L-embedded Banach spaces, not only separable ones.

Theorem The L-projection of an L-embedded Banach space sends relatively $w^{*}$ sequentially compact sets into relatively weakly sequentially compact sets.

Proof. We recall that a series $\sum z_{j}$ in a Banach space $Z$ is called weakly unconditionally Cauchy (wuC for short) if $\sum\left|z^{*}\left(z_{j}\right)\right|$ converges for each $z^{*} \in Z^{*}$ or, 
equivalently, if there is a number $M$ such that $\left\|\sum_{j=1}^{n} \alpha_{j} z_{j}\right\| \leq M \max _{1 \leq j \leq n}\left|\alpha_{j}\right|$ for all $n \in \mathbb{N}$ and all scalars $\alpha_{j}$.

Let $X$ be an L-embedded Banach space with L-projection $P$. We have the decomposition $X^{* *}=X \oplus_{1} X_{\mathrm{s}}$ where $X_{\mathrm{s}}$ denotes the range of the projection $Q=\operatorname{id}_{X^{* *}}-P$. Let $\left(x_{n}\right)$ be a sequence in $X$ and let $\left(t_{n}\right)$ be a sequence in $X_{\mathrm{s}}$. Further, consider a cluster point $x+x_{\mathrm{s}}$ of the $x_{n}$ in the $w^{*}$-topology of $X^{* *}$ (with $x \in X, x_{\mathrm{s}} \in X_{\mathrm{s}}$ ).

Claim Given $x^{*} \in X^{*}$ of norm one there are a wuC-series $\sum x_{k}^{*}$ in $X^{*}$ and a sequence $\left(n_{k}\right)$ in $\mathbb{N}$ such that, for all $k \in \mathbb{N}$,

$$
\begin{aligned}
t_{n_{k}}\left(x_{k}^{*}\right) & =0 \\
\lim _{k} x_{k}^{*}\left(x_{n_{k}}\right) & =x_{\mathrm{s}}\left(x^{*}\right) .
\end{aligned}
$$

Proof of the claim: Let $1>\varepsilon>0$ and let $\left(\varepsilon_{j}\right)$ be a sequence of numbers decreasing to zero such that $0<\varepsilon_{j}<1$ and $\prod_{j=1}^{\infty}\left(1+\varepsilon_{j}\right)<1+\varepsilon$.

By induction over $k \in \mathbb{N}_{0}=\mathbb{N} \cup\{0\}$ we shall construct two sequences $\left(x_{k}^{*}\right)_{k \in \mathbb{N}_{0}}$ and $\left(y_{k}^{*}\right)_{k \in \mathbb{N}_{0}}$ in $X^{*}$ (of which the first members $x_{0}^{*}$ and $y_{0}^{*}$ are auxiliary elements used only for the induction) and an increasing sequence $\left(n_{k}\right)$ of indices such that, for all (real or complex) scalars $\alpha_{j}$ and with $\beta=x_{\mathrm{s}}\left(x^{*}\right)$, the following hold:

$$
\begin{aligned}
& x_{0}^{*}=0,\left\|y_{0}^{*}\right\|=1, \\
&\left\|\alpha_{0} y_{k}^{*}+\sum_{j=1}^{k} \alpha_{j} x_{j}^{*}\right\| \leq\left(\prod_{j=1}^{k}\left(1+\varepsilon_{j}\right)\right) \max _{0 \leq j \leq k}\left|\alpha_{j}\right|, \quad \text { if } k \geq 1, \\
& t_{n_{k}}\left(x_{k}^{*}\right)=0 \\
& y_{k}^{*}(x)=0, \quad \text { and } \quad x_{\mathrm{s}}\left(y_{k}^{*}\right)=\beta, \\
&\left|x_{k}^{*}\left(x_{n_{k}}\right)-\beta\right|<\varepsilon_{k} .
\end{aligned}
$$

We set $n_{0}=1, x_{0}^{*}=0$ and $y_{0}^{*}=x^{*}$.

For the following it is useful to recall some properties of $P$ : The restriction of $P^{*}$ to $X^{*}$ is an isometric isomorphism from $X^{*}$ onto $X_{\mathrm{s}}^{\perp}$ with $\left(P^{*} y^{*}\right)_{\mid X}=y^{*}$ for all $y^{*} \in X^{*}, Q$ is a contractive projection and $X^{* * *}=X_{\mathrm{s}}^{\perp} \oplus_{\infty} X^{\perp}$ (where $X^{\perp}$ is the annihilator of $X$ in $\left.X^{* * *}\right)$.

For the induction step suppose now that $x_{0}^{*}, \ldots, x_{k}^{*}, y_{0}^{*}, \ldots, y_{k}^{*}$ and $n_{0}, \ldots, n_{k}$ have been constructed and satisfy conditions $(0.3)-(0.7)$. Since $x+x_{\mathrm{s}}$ is a $w^{*}$-cluster point of the $x_{n}$ there is an index $n_{k+1}$ such that

$$
\left|x_{\mathrm{s}}\left(y_{k}^{*}\right)-y_{k}^{*}\left(x_{n_{k+1}}-x\right)\right|<\varepsilon_{k+1} .
$$

Put

$$
\begin{aligned}
& E=\operatorname{lin}\left(\left\{x^{*}, x_{0}^{*}, \ldots, x_{k}^{*}, y_{k}^{*}, P^{*} x_{0}^{*}, \ldots, P^{*} x_{k}^{*}, P^{*} y_{k}^{*}\right\}\right) \subset X^{* * *}, \\
& F=\operatorname{lin}\left(\left\{x_{n_{k+1}}, t_{n_{k+1}}, x, x_{\mathrm{s}}\right\}\right) \subset X^{* *} .
\end{aligned}
$$


Clearly $Q^{*} x_{j}^{*}, Q^{*} y_{k}^{*} \in E$ for $0 \leq j \leq k$. By the principle of local reflexivity there is an operator $R: E \rightarrow X^{*}$ such that

$$
\begin{aligned}
\left(1-\varepsilon_{k+1}\right)\left\|e^{* * *}\right\| & \leq\left\|R e^{* * *}\right\| \leq\left(1+\varepsilon_{k+1}\right)\left\|e^{* * *}\right\|, \\
f^{* *}\left(R e^{* * *}\right) & =e^{* * *}\left(f^{* *}\right), \\
R_{\mid E \cap X^{*}} & =\operatorname{id}_{E \cap X^{*}}
\end{aligned}
$$

for all $e^{* * *} \in E$ and $f^{* *} \in F$.

We define

$$
x_{k+1}^{*}=R P^{*} y_{k}^{*} \quad \text { and } \quad y_{k+1}^{*}=R Q^{*} y_{k}^{*} .
$$

In the following we use the convention $\sum_{j=1}^{0}(\cdots)=0$. Then we have that

$$
\alpha_{0} y_{k+1}^{*}+\sum_{j=1}^{k+1} \alpha_{j} x_{j}^{*}=R\left(Q^{*}\left(\alpha_{0} y_{k}^{*}+\sum_{j=1}^{k} \alpha_{j} x_{j}^{*}\right)+P^{*}\left(\alpha_{k+1} y_{k}^{*}+\sum_{j=1}^{k} \alpha_{j} x_{j}^{*}\right)\right) .
$$

Now (0.4) (for $k+1$ instead of $k$ ) can be seen as follows:

$$
\begin{aligned}
& \left\|\alpha_{0} y_{k+1}^{*}+\sum_{j=1}^{k+1} \alpha_{j} x_{j}^{*}\right\| \leq \\
& \stackrel{(0.9)}{\leq}\left(1+\varepsilon_{k+1}\right)\left\|Q^{*}\left(\alpha_{0} y_{k}^{*}+\sum_{j=1}^{k} \alpha_{j} x_{j}^{*}\right)+P^{*}\left(\alpha_{k+1} y_{k}^{*}+\sum_{j=1}^{k} \alpha_{j} x_{j}^{*}\right)\right\| \\
& =\left(1+\varepsilon_{k+1}\right) \max \left\{\left\|Q^{*}\left(\alpha_{0} y_{k}^{*}+\sum_{j=1}^{k} \alpha_{j} x_{j}^{*}\right)\right\|,\left\|P^{*}\left(\alpha_{k+1} y_{k}^{*}+\sum_{j=1}^{k} \alpha_{j} x_{j}^{*}\right)\right\|\right\} \\
& \leq \quad\left(1+\varepsilon_{k+1}\right) \max \left\{\left\|\alpha_{0} y_{k}^{*}+\sum_{j=1}^{k} \alpha_{j} x_{j}^{*}\right\|,\left\|\alpha_{k+1} y_{k}^{*}+\sum_{j=1}^{k} \alpha_{j} x_{j}^{*}\right\|\right\} \\
& \leq \quad\left(\prod_{j=1}^{k+1}\left(1+\varepsilon_{j}\right)\right) \max \left\{\max _{0 \leq j \leq k}\left|\alpha_{j}\right|, \max _{1 \leq j \leq k+1}\left|\alpha_{j}\right|\right\} \\
& =\left(\prod_{j=1}^{k+1}\left(1+\varepsilon_{j}\right)\right) \max _{0 \leq j \leq k+1}\left|\alpha_{j}\right|
\end{aligned}
$$

where the last inequality comes from (0.3) if $k=0$, and from (0.4), if $k \geq 1$.

The conditions (0.5) and (0.6) (for $k+1$ instead of $k$ ) are easy to verify because $P t_{n_{k+1}}=0, Q x=0$ and $Q x_{\mathrm{s}}=0$ thus, by $(0.10)$

$$
\begin{aligned}
& t_{n_{k+1}}\left(x_{k+1}^{*}\right)=y_{k}^{*}\left(P t_{n_{k+1}}\right)=0, \\
& y_{k+1}^{*}(x)=y_{k}^{*}(Q x)=0 \quad \text { and } \quad x_{\mathrm{s}}\left(y_{k+1}^{*}\right)=Q^{*} y_{k}^{*}\left(x_{\mathrm{s}}\right)=x_{\mathrm{s}}\left(y_{k}^{*}\right)=\beta \text {. }
\end{aligned}
$$

Finally, we have

$$
x_{k+1}^{*}\left(x_{n_{k+1}}\right)-\beta=y_{k}^{*}\left(x_{n_{k+1}}\right)-\beta=y_{k}^{*}\left(x_{n_{k+1}}-x\right)-x_{\mathrm{s}}\left(y_{k}^{*}\right)
$$


by (0.6) whence $(0.7)$ for $k+1$ by $(0.8)$. This ends the induction and proves the CLAIM.

Put now $x_{n}^{* *}=x_{n}+t_{n}$ and suppose that $\left(x_{n}^{* *}\right)$ is $w^{*}$-null. We define a sequence $\left(\mu_{n}\right)$ of finitely additive measures on the subsets of $\mathbb{N}$ by $\mu_{n}(A)=x_{n}^{* *}\left(\sum_{k \in A} x_{k}^{*}\right)$ for all $A \subset \mathbb{N}$ where $\sum_{k \in A} x_{k}^{*}$ is to be understood in the $w^{*}$-topology of $X^{*}$. Then $\mu_{n}(A) \rightarrow 0$ for all $A \subset \mathbb{N}$ and by (0.1) and Phillips' original lemma ([8], [1, p. 83]) we get

$$
\left|x_{k}^{*}\left(x_{n_{k}}\right)\right|=\left|x_{n_{k}}^{* *}\left(x_{k}^{*}\right)\right| \leq \sum_{j}\left|x_{n_{k}}^{* *}\left(x_{j}^{*}\right)\right|=\sum_{j}\left|\mu_{n_{k}}(\{j\})\right| \rightarrow 0 .
$$

Thus $x_{\mathrm{s}}\left(x^{*}\right)=0$ by (0.2). Since $x^{*}$ was an arbitrary normalized element this means that $x_{\mathrm{s}}=0$. It follows that the $w^{*}$-closure in $X^{* *}$ of the set consisting of the $x_{n}$ lies in $X$. Hence this set is relatively weakly compact (or, equivalently, relatively weakly sequentially compact). This proves the theorem.

It is not clear whether the L-projection is actually $w^{*}$-weak-continuous. In two cases this happens trivially: firstly if the dual $X^{*}$ is a Grothendick space (which by definition means that in $X^{* *} w^{*}$-convergent sequences converge weakly) and secondly if $X$ is the dual of an M-embedded Banach space $Y$ (see [3, Ch. III]) because in this case $P$ is the canonical restriction projection from $Y^{* * *}$ onto $Y^{*}([3$, Prop. III.2.4]) and the $x_{n}$ tend to 0 on the elements of $Y$ and thus any weak cluster point $x$ of $\left(x_{n}\right)$ is necessarily zero. Alternatively, in the latter case the result $[3$, Prop. III.3.6], which was mentionned above, applies because M-embedded Banach spaces have property (V) ([2] or [3, Th. III.3.4]).

\section{References}

[1] J. Diestel. Sequences and Series in Banach Spaces. Springer, Berlin-Heidelberg-New York, 1984.

[2] G. Godefroy and P. Saab. Weakly unconditionally convergent series in $M$-ideals. Math. Scand., 64:307-318, 1989.

[3] P. Harmand, D. Werner, and W. Werner. M-ideals in Banach Spaces and Banach Algebras. Lecture Notes in Mathematics 1547. Springer, 1993.

[4] W. B. Johnson and J. Lindenstrauss. Handbook of the Geometry of Banach Spaces, Volumes 1 and 2. North Holland, 2001, 2003.

[5] P. K. Lin. Köthe-Bochner function spaces. Birkäuser, Boston, 2004.

[6] J. Lindenstrauss and L. Tzafriri. Classical Banach Spaces I and II. Springer, BerlinHeidelberg-New York, 1977, 1979.

[7] H. Pfitzner. Separable L-embedded Banach spaces are unique preduals. Bull. Lond. Math. Soc., 39:1039-1044, 2007.

[8] R. S. Phillips. On linear transformations. Trans. Amer. Math. Soc., 48:516-541, 1940.

[9] N. Randrianantoanina. Complemented copies of $\ell^{1}$ and Peczyski's property $\left(V^{*}\right)$ in Bochner function spaces. Canadian J. Math., 48:625-640, 1996. 
[10] M. Talagrand. Weak Cauchy sequences in $L^{1}(E)$. Amer. J. Math., 106:703-724, 1984.

[11] A. Ülger. The weak Phillips property. Colloq. Math., 87:147-158, 2001.

Hermann Pfitzner

Université d'Orléans, BP 6759, F-45067 Orléans Cedex 2, France

e-mail: hermann.pfitzner@univ-orleans.fr 